\title{
Identificação Macroscópica e Microscópica das Madeiras do Parque Nacional do Itatiaia: Chave Interativa Desenvolvida em Software Livre
}

\author{
Thaís Siston ${ }^{1}$, Arno Fritz das Neves Brandes ${ }^{2}$, Leonardo Bona ${ }^{1}$, Gláucia Crispim ${ }^{1}$, Alessandra Voigt ${ }^{1} \&$ Claudia Franca Barros $^{1}$
}

Recebido em 22/10/2019 - Aceito em 30/03/2020

1 Instituto de Pesquisas Jardim Botânico do Rio de Janeiro/JBRJ, Rio de Janeiro/RJ, Brasil. CEP: 22.460-030. <tsiston@yahoo.com.br,
bona.nascimento@gmail.com, glaucia.crispim@yahoo.com.br, arvoigt@gmail.com, cbarros@jbrj.gov.br>.
2 Universidade Federal Fluminense. <arnofritz@id.uff>.

RESUMO - O Parque Nacional do Itatiaia (PARNA do Itatiaia), mais antigo do Brasil, foi criado em 1937, e sua relação com o Instituto de Pesquisas Jardim Botânico do Rio de Janeiro é bastante antiga, com muitas pesquisas em desenvolvimento nas mais diferentes áreas de atuação da botânica - dentre elas, as pesquisas em anatomia da madeira. Este trabalho se propôs a estudar a macro e microscopia de madeiras de árvores desta unidade de conservação, visando à criação de chaves interativas em software livre. Foram descritas treze espécies: Cabralea canjerana, Clethra scabra var. reticulata, Croton floribundus, Croton vulnerarius, Dalbergia variabilis, Guatteria candolleana, Jacaranda caroba, Leucochloron incuriale, Lonchocarpus sericeus, Machaerium nyctitans, Peltophorum vogelianum, Senna multijuga e Vitex taruma. Algumas descrições foram adicionadas, sendo outras oriundas da base de espécies do Laboratório de Botânica Estrutural do Instituto de Pesquisas Jardim Botânico do Rio de Janeiro, totalizando 41 espécies para a chave macroscópica e 61 espécies para a chave microscópica. As descrições anatômicas seguiram a metodologia usual para estudos dessa natureza. As chaves interativas foram desenvolvidas no software Xper3 e contaram com a inserção de 106 caracteres macroscópicos e 221 caracteres microscópicos, devidamente ilustrados e hospedados no site da Universidade Federal Fluminense (UFF), disponível em: < http://gbg.sites.uff.br/lamad/> . Essas chaves são as primeiras publicadas para o bioma Mata Atlântica e permitirão, com a identificação das árvores do PARNA do Itatiaia a partir de sua madeira, divulgar a diversidade de árvores da unidade de conservação e formar recursos humanos em biodiversidade.

Palavras-chave: Xper3; anatomia da madeira; unidade de conservação.

\section{Macroscopic and Microscopic Wood Identification of Itatiaia National Park: Interactive Key Developed in Free Software}

\begin{abstract}
Parque Nacional do Itatiaia (PARNA do Itatiaia), the oldest in Brazil, was created in 1937 and its relationship with the Instituto de Pesquisas Jardim Botânico do Rio de Janeiro is quite old, with much research being carried out in the most diverse areas of botany. Among them, the research in wood anatomy. This work aims to study the macro and microscopy of tree woods of this conservation unit aiming at the creation of interactive keys in free software. Thirteen species were described: Cabralea canjerana, Clethra scabra var. reticulata, Croton floribundus, Croton vulnerarius, Dalbergia variabilis, Guatteria candolleana, Jacaranda caroba, Leucochloron incuriale, Lonchocarpus sericeus, Machaerium nyctitans, Peltophorum vogelianum, Senna multijuga and Vitex taruma. To these descriptions were added other from the species base of the Laboratório de Botânica Estrutural do Instituto de Pesquisas Jardim Botânico do Rio de Janeiro totaling 41 species for the macroscopic key and 61 species for the microscopic key. The anatomical descriptions followed the usual methods for studies of this nature. The interactive keys were developed in the Xper3 software and included 106 macroscopic characters and 221 microscopic characters, illustrated and hosted at the Universidade Federal Fluminense (UFF) website, available at: < http: //gbg.sites.uff. br / lamad / $>$. These keys are the first published for the Atlantic Forest biome and will allow the identification of PARNA do Itatiaia trees from their wood, disseminate the diversity of trees of the conservation unit and the education in biodiversity.
\end{abstract}

Keywords: Xper3; wood anatomic; protected areas. 


\title{
Identificación de Madera Macroscópica y Microscópica del Parque Nacional de Itatiaia: Clave Interactiva Desarrollada en Software Libre
}

\begin{abstract}
RESUMEN - El Parque Nacional Itatiaia (PARNA do Itatiaia), el más antiguo de Brasil, se estableció en 1937 y su relación con el Instituto de Pesquisas Jardim Botânico do Rio de Janeiro es bastante antigua, con investigaciones en las áreas más diversas, entre ellas anatomía de la madera. Este trabajo tuvo como objetivo estudiar la macro y la microscopía de las maderas de árboles de esta unidad de conservación con el fin de crear claves interactivas en un software libre. Se describieron trece espécies: Cabralea canjerana, Clethra scabra var. reticulata, Croton floribundus, Croton vulnerarius, Dalbergia variabilis, Guatteria candolleana, Caroba jacaranda, Leucochloron incuriale, Lonchocarpus sericeus, Machaerium nyctitans, Peltophorum vogelianum, Senna multijuga y Vitex taruma. A estas descripciones se añadieron otras especies del Laboratório de Botânica Estrutural do Instituto de Pesquisas Jardim Botânico do Rio de Janeiro, con un total de 41 especies para la clave macroscópica y 61 especies para la clave microscópica. Las descripciones anatómicas siguieron los métodos habituales para estudios de esta naturaleza. Las claves interactivas se desarrollaron en el software Xper3 e incluyeron la inserción de 106 caracteres macroscópicos y 221 caracteres microscópicos, debidamente ilustrados y alojados en el sitio web de la Universidade Federal Fluminense (UFF), disponible en: <http: //gbg.sites.uff. br / lamad />. Estas claves son las primeras publicadas para el bioma del Bosque Atlántico y permitirán identificar los árboles del PARNA de Itatiaia a através de la anatomia de la madera, difundir la diversidad de árboles de la unidad de conservación y la formación de recursos humanos en biodiversidad.
\end{abstract}

Palabras clave: Xper3; anatomía de madera, áreas protegidas.

\section{Introdução}

O bioma Mata Atlântica é definido pela Lei $\mathrm{n}^{\circ}$ 11.428/2006 como: "formações florestais nativas e ecossistemas associados, com as respectivas delimitações estabelecidas em mapa do Instituto Brasileiro de Geografia e Estatística (IBGE), conforme regulamento: floresta ombrófila densa; floresta ombrófila mista, também denominada de mata de araucárias; floresta ombrófila aberta; floresta estacional semidecidual; e floresta estacional decidual, bem como os manguezais, as vegetações de restingas, campos de altitude, brejos interioranos e encraves florestais do Nordeste". Inicialmente, sua área abrangia, aproximadamente, $1.300 .000 \mathrm{~km} 2$, em 17 estados brasileiros e em áreas no interior do Paraguai e da Argentina (Campanili \& Schaffer 2010, Ribeiro et al. 2011, Scarano \& Ceotto 2015). Atualmente o bioma encontra-se em estado crítico de fragmentação, devido à intensa ocupação $e$ ao desenvolvimento de atividades humanas, possuindo áreas remanescentes com cerca de $15,2 \%$ da cobertura original (Fundação SOS Mata Atlântica e Instituto Nacional de Pesquisas Espaciais 2018). Devido a essa fragmentação e suas altas taxas de biodiversidade - e por possuir $40 \%$ das espécies endêmicas de todo o Brasil -, o bioma é um dos 35 hotspots mundiais de biodiversidade (Myers 2000, Mittermaier et al. 2005), abrigando cerca de 46.485 espécies reconhecidas para a flora do Brasil e possuindo $60 \%$ de toda a lista de espécies ameaçadas da flora brasileira (Forzza et al. 2012, Flora do Brasil 2020 em construção, Ministério do Meio Ambiente 2008).

O Brasil possui 304 unidades de conservação (UCs) federais, das quais 78 estão inseridas no bioma Mata Atlântica, correspondendo a $25,7 \%$ do total. Na região sudeste brasileira, existem $45 \mathrm{UCs}$ federais, que englobam, em sua maioria, fragmentos de Mata Atlântica (ICMBio 2014a).

O Parque Nacional do Itatiaia (PARNA Itatiaia) foi a primeira UC a ser criada oficialmente em território nacional, em 14 de junho de 1937, através do Decreto $\mathrm{n}^{\circ} 1.713$, e, após 45 anos, o Decreto $n^{\circ} 87.586$ ampliou sua área de abrangência, tendo como missão: a preservação dos ecossistemas naturais - relevância ecológica e beleza cênica; realização de pesquisas científicas em diversas áreas do conhecimento; desenvolvimento de atividades socioambientais -, educação e interpretação ambiental, turismo ecológico e recreação em contato com a natureza (Rodrigues et al. 2013, CNUC/MMA 2014). Atualmente, possui uma área de 28.084,10 hectares no bioma Mata Atlântica dos estados do Rio de Janeiro (municípios Itatiaia e Resende) e Minas Gerais (municípios Bocaina de Minas e 
Itamonte), integrando o Mosaico de UCs da Serra da Mantiqueira e o Corredor Ecológico da Serra da Mantiqueira, que fazem parte da Reserva da Biosfera da Mata Atlântica, reconhecidos pela UNESCO (Rodrigues et al. 2013). O maciço do Itatiaia serve de divisor das bacias do rio Paraíba e do rio Grande, afluente do rio Paraná. O PARNA Itatiaia possui relevo bastante variado, composto por cadeias montanhosas que variam de 600 a 2.791 metros, as quais são de grande importância geológica, por abrigar rochas pouco comuns para o território brasileiro. Essa variação altitudinal e no relevo propiciam uma grande variedade de ecossistemas e uma biodiversidade regional característica (CNUC/MMA 2014, ICMBiob 2014, Rodrigues et al. 2013). Uma das primeiras ações para a conservação da região do Itatiaia foi a criação de uma reserva florestal em 1914. Em 1929, após uma campanha visando à preservação da área pelos pesquisadores viajantes do Jardim Botânico do Rio de Janeiro (JBRJ), a reserva se tornou uma estação biológica gerida pelo JBRJ, cujos objetivos foram preservar a área, que já sofria ações de degradação; dar continuidade às pesquisas científicas; e coletar espécimes botânicos, zoológicos e geológicos. Até a data de criação do PARNA Itatiaia, os pesquisadores do JBRJ foram os principais responsáveis por pesquisas e publicações (Jardim Botânico do Rio de Janeiro 2008, Rodrigues et al. 2013), e o interesse científico permanece ao longo dos anos (p.ex.: Silva Neto \& Ávila Júnior 2007, Brandes \& Barros 2008, Brandes et al. 2011, Wolowski \& Freitas 2015).

Dentre as pesquisas realizadas no PARNA Itatiaia, merecem destaque os estudos relacionados às árvores, em especial à anatomia da madeira. Essa linha de pesquisa é muito utilizada para a identificação de amostras vegetais sem a parte reprodutiva. Para isso, inúmeras publicações, manuais e chaves de identificação foram confeccionados no século XX para facilitar o trabalho dos anatomistas da madeira (Record \& Hess 1944, Record 1944, Mainieri 1958). No século XXI, o conhecimento dessas publicações vem sendo transferido para bases de dados $e$ chaves interativas para identificação, como as encontradas nas publicações de Richter et al. (2002): identificação de madeiras comerciais de diversos países baseada em caracteres observáveis com microscópios; Coradin et al. (2010): para a identificação de madeiras comerciais do Brasil baseada em caracteres observáveis a olhos desarmados e lupa com aumento de 10x; e Kock et al. (2011): com o desenvolvimento de chave eletrônica no software Delta com caracteres observáveis com microscópico para identificação de madeiras comerciais listadas na CITES.

Diante da importância do PARNA Itatiaia como a mais antiga UC brasileira, e por preservar a rica biodiversidade da Mata Atlântica fluminense, o objetivo deste estudo foi analisar e descrever, macroscopicamente e microscopicamente, as amostras de madeira de espécies provenientes do PARNA Itatiaia depositadas na Xiloteca do Instituto de Pesquisas Jardim Botânico do Rio de Janeiro $(\mathrm{RBw})$, e desenvolver chaves interativas para identificação das espécies a partir de caracteres macroscópicos e microscópicos da madeira.

\section{Material e Métodos}

Amostras de madeira de 13 espécies provenientes do PARNA Itatiaia e tombadas no $\mathrm{RBw}$ foram analisadas (Tabela 1).

Tabela 1 - Espécies presentes nas chaves interativas de identificação. Espécies descritas na base de dados do LBE (*); espécies descritas no trabalho atual (**); e espécies descritas por Barros et al. (2008) (***). RBw: Xiloteca do Jardim Botânico do Rio de Janeiro.

\begin{tabular}{|l|l|c|c|c|}
\hline \multicolumn{1}{c|}{ Família } & \multicolumn{1}{c|}{ Espécie } & RBw & \multicolumn{2}{c|}{ Chave } \\
\cline { 2 - 6 } & & \multicolumn{1}{c|}{ Macro. } & Micro. \\
\hline ANNONACEAE & Guatteria candolleana Schltdl. ** & 2249 & X & X \\
\hline BIGNONIACEAE & Jacaranda caroba DC. $* *$ & 2225 & X & X \\
\hline CLETHRACEAE & Clethra scabra var. reticulata (Meisn.) Sleumer ** & 2233 & X & X \\
\hline
\end{tabular}




\begin{tabular}{|c|c|c|c|c|}
\hline \multirow{2}{*}{ EUPHORBIACEAE } & Croton floribundus Spreng. ** & 2229 & $\mathrm{x}$ & $\mathrm{X}$ \\
\hline & Croton vulnerarius Baill. ** & 2259 & $\mathrm{x}$ & $\mathrm{X}$ \\
\hline \multirow{6}{*}{ FABACEAE } & Dalbergia variabilis Vogel ** & 2223 & $\mathrm{x}$ & $\mathrm{X}$ \\
\hline & Leucochloron incuriale (Vell.) Barneby \& J.W. Grimes ** & 2218 & $\mathrm{x}$ & $\mathrm{x}$ \\
\hline & Lonchocarpus sericeus (Poir.) Kunth ex DC. ** & 2319 & $\mathrm{x}$ & $\mathrm{X}$ \\
\hline & Machaerium nyctitans (Vell.) Benth. ** & 2240 & $\mathrm{x}$ & $\mathrm{X}$ \\
\hline & Peltophorum vogelianum Benth. ** & 2234 & $\mathrm{x}$ & $\mathrm{X}$ \\
\hline & Senna multijuga (Rich.) H.S.Irwin \& Barneby ** & 2247 & $\mathrm{x}$ & $\mathrm{X}$ \\
\hline LAMIACEAE & Vitex taruma Mart. ** & 2219 & $\mathrm{x}$ & $\mathrm{X}$ \\
\hline MELIACEAE & Cabralea canjerana (Vell.) Mart. ** & 2253 & $\mathrm{x}$ & $\mathrm{X}$ \\
\hline \multirow{5}{*}{ APOCYNACEAE } & \multirow{5}{*}{ Aspidosperma polyneuron Müll. Arg.* } & 7782 & \multirow{5}{*}{$\mathrm{x}$} & \multirow{5}{*}{$\mathrm{X}$} \\
\hline & & 7820 & & \\
\hline & & 7821 & & \\
\hline & & 7828 & & \\
\hline & & 7830 & & \\
\hline \multirow{5}{*}{ AQUIFOLIACEAE } & \multirow{3}{*}{ Ilex chamaedryfolia Reissek * } & 8782 & & \multirow{3}{*}{$\mathrm{x}$} \\
\hline & & 8887 & & \\
\hline & & 8891 & & \\
\hline & \multirow{2}{*}{ Ilex theezans Mart. * } & 880 & & \multirow{2}{*}{$\mathrm{X}$} \\
\hline & & 8810 & & \\
\hline ASTERACEAE & Vernonia discolor (Spreng.) Less. * & 8827 & & $\mathrm{X}$ \\
\hline CELASTRACEAE & $\begin{array}{l}\text { Monteverdia dasyclada (Mart.) Biral Biral (Mart.) } \\
\text { Mart. }\end{array}$ & 8917 & & $\mathrm{X}$ \\
\hline \multirow{2}{*}{ CHRYSOBALANACEAE } & Hirtella hebeclada Moric. $* * * *$ & $\begin{array}{l}7575 \\
7577 \\
7616 \\
7622 \\
7773\end{array}$ & $\mathrm{x}$ & $\mathrm{x}$ \\
\hline & Licania krunthiana Hook. f. */*** & $\begin{array}{l}7604 \\
7605 \\
7609 \\
7767\end{array}$ & $\mathrm{x}$ & $\mathrm{X}$ \\
\hline \multirow[b]{2}{*}{ CUNONIACEAE } & Weinmannia discolor Gardner * & 8809 & & $\mathrm{X}$ \\
\hline & Weinmannia organensis Gardner * & $\begin{array}{l}8837 \\
8841\end{array}$ & & $\mathrm{x}$ \\
\hline ELAEOCARPACEAE & Sloanea obtusifolia (Moric.) K.Schm. **** & 7710 & & $\mathrm{X}$ \\
\hline
\end{tabular}




\begin{tabular}{|c|c|c|c|c|}
\hline \multirow{2}{*}{ EUPHORBIACEAE } & Actinostemon concolor (Spreng.) Müll. Arg. */*** & $\begin{array}{l}7708 \\
7756 \\
7758 \\
7774 \\
7776\end{array}$ & X & $\mathrm{X}$ \\
\hline & Pachystroma longifolium (Nees) I. M. Johnst ***** & $\begin{array}{l}7628 \\
7631 \\
7712 \\
7752 \\
7839\end{array}$ & $\mathrm{x}$ & $\mathrm{X}$ \\
\hline \multirow{5}{*}{ FABACEAE } & Ormosia friburgensis Taub. ex Glaz. ****** & $\begin{array}{l}7704 \\
7603 \\
7764 \\
7788\end{array}$ & X & $\mathrm{X}$ \\
\hline & Pseudopiptadenia leptostachya (Benth.) Rauschert */*** & $\begin{array}{l}7584 \\
7619 \\
7624 \\
7626 \\
7787\end{array}$ & $\mathrm{X}$ & $\mathrm{X}$ \\
\hline & Pterocarpus rohrii Vahl * & $\begin{array}{l}7697 \\
7613 \\
7625 \\
7771 \\
7783\end{array}$ & X & $\mathrm{X}$ \\
\hline & Zollernia glabra (Spreng.) Yakovlev * & $\begin{array}{l}7589 \\
7595 \\
7750 \\
7755 \\
7777\end{array}$ & $\mathrm{X}$ & $\mathrm{X}$ \\
\hline & Zollernia ilicifolia (Brongn.) Vogel * & $\begin{array}{l}7627 \\
7765 \\
7827\end{array}$ & $\mathrm{X}$ & $\mathrm{X}$ \\
\hline \multirow{4}{*}{ LAURACEAE } & Ocotea itatiaiae Vattimo-Gil */*** & $\begin{array}{l}7583 \\
7593 \\
7751 \\
7761 \\
7772\end{array}$ & $\mathrm{X}$ & $\mathrm{X}$ \\
\hline & Ocotea odorifera (Vellozo) Rohwer ***** & $\begin{array}{l}7572 \\
7711 \\
7745\end{array}$ & $\mathrm{X}$ & $\mathrm{X}$ \\
\hline & Ocotea tenuiflora (Nees) Mez ****** & $\begin{array}{l}7599 \\
7629 \\
7798\end{array}$ & $\mathrm{X}$ & $\mathrm{X}$ \\
\hline & $\begin{array}{l}\text { Rhodostemonodaphne macrocalyx (Meisn.) Rohwer ex } \\
\text { Madriñán */*** }\end{array}$ & $\begin{array}{l}7588 \\
7592 \\
7602\end{array}$ & $\mathrm{X}$ & $\mathrm{X}$ \\
\hline
\end{tabular}




\begin{tabular}{|c|c|c|c|c|}
\hline \multirow{3}{*}{ MELASTOMATACEAE } & Miconia cinerascens Miq.* & $\begin{array}{l}8773 \\
8924\end{array}$ & & $\mathrm{X}$ \\
\hline & Miconia pusilliflora (DC.) Naudin* & 8876 & & $\mathrm{X}$ \\
\hline & Miconia sellowiana Naudin* & $\begin{array}{l}8805 \\
8773\end{array}$ & & $\mathrm{X}$ \\
\hline MYRISTICACEAE & Virola oleifera (Schott) A.C.Sm */*** & $\begin{array}{l}7618 \\
7717 \\
7775 \\
8393\end{array}$ & $\mathrm{X}$ & $\mathrm{x}$ \\
\hline \multirow{8}{*}{ MYRTACEAE } & Eugenia cachoeirensis O. Berg */*** & $\begin{array}{l}7580 \\
7586 \\
7715 \\
7754\end{array}$ & $\mathrm{x}$ & $\mathrm{X}$ \\
\hline & Eugenia chlorophylla O. Berg */*** & $\begin{array}{l}7611 \\
7747 \\
7757\end{array}$ & $\mathrm{x}$ & $\mathrm{x}$ \\
\hline & Eugenia hiemalis Cambess. */*** & $\begin{array}{l}7587 \\
7714\end{array}$ & $\mathrm{x}$ & $\mathrm{X}$ \\
\hline & Myrceugenia myrcioides (Cambess.) O.Berg * & 8845 & & $\mathrm{X}$ \\
\hline & Myrceugenia ovata var. acutata (D.Legrand) Landrum * & 8853 & & $\mathrm{X}$ \\
\hline & Myrcia breviramis (O.Berg) D.Legrand * & $\begin{array}{l}8801 \\
8808 \\
8829 \\
8865 \\
8890\end{array}$ & & $\mathrm{X}$ \\
\hline & Myrcia richardiana (O.Berg) Kiaersk. * & 8814 & & $\mathrm{X}$ \\
\hline & Siphoneugena kuhlmannii Mattos * & $\begin{array}{l}8793 \\
8896 \\
8772\end{array}$ & & $\mathrm{X}$ \\
\hline \multirow[b]{2}{*}{ PRIMULACEAE } & Myrsine coriacea (Sw.) R.Br. ex Roem. \& Schult. * & 8839 & & $\mathrm{X}$ \\
\hline & Myrsine gardneriana A.DC. * & $\begin{array}{l}8768 \\
8815 \\
8875 \\
8945\end{array}$ & & $\mathrm{X}$ \\
\hline \multirow{4}{*}{ RUBIACEAE } & Schizocalyx cuspidatus (A.St.-Hil.) Kainul. \& B.Bremer */**** & $\begin{array}{l}7769 \\
7785 \\
8373 \\
8374\end{array}$ & $\mathrm{x}$ & $\mathrm{X}$ \\
\hline & Coussarea congestiflora Müll. Arg. */**** & $\begin{array}{l}7597 \\
8376 \\
8380\end{array}$ & $\mathrm{x}$ & $\mathrm{X}$ \\
\hline & Coutarea hexandra (Jacq.) K.Schum. */*** & $\begin{array}{l}7576 \\
7585 \\
8375 \\
8379\end{array}$ & $\mathrm{x}$ & $\mathrm{X}$ \\
\hline & Psychotria vellosiana Benth. * & 8941 & & $\mathrm{X}$ \\
\hline
\end{tabular}




\begin{tabular}{|c|c|c|c|c|}
\hline SALICACEAE & Xylosma prockia (Turcz.) Turcz. ***** & $\begin{array}{l}7574 \\
7762\end{array}$ & $\mathrm{x}$ & $\mathrm{X}$ \\
\hline SAPINDACEAE & Cupania oblongifolia Mart. */*** & $\begin{array}{l}7829 \\
7581 \\
7831\end{array}$ & $\mathrm{x}$ & $\mathrm{X}$ \\
\hline \multirow{3}{*}{ SAPOTACEAE } & Diploon cuspidatum (Hoehne) Cronquist ****** & $\begin{array}{l}7620 \\
7621 \\
7779\end{array}$ & $\mathrm{x}$ & $\mathrm{X}$ \\
\hline & $\begin{array}{l}\text { Micropholis crassipedicellata (Mart. \& Eichler ex Miq) Pierre } \\
* / * * * *\end{array}$ & $\begin{array}{l}7594 \\
7598 \\
7713 \\
7709 \\
7753\end{array}$ & $\mathrm{x}$ & $\mathrm{X}$ \\
\hline & Pouteria torta (Mart.) Raldk. */*** & $\begin{array}{l}7759 \\
7780 \\
8397\end{array}$ & $\mathrm{x}$ & $\mathrm{X}$ \\
\hline SYMPLOCACEAE & Symplocos densiflora Brand & 8767 & & $\mathrm{X}$ \\
\hline URTICACEAE & Coussapoa microcarpa (Schott) Rizzini */*** & $\begin{array}{l}7578 \\
7608 \\
7617 \\
7786\end{array}$ & $\mathrm{X}$ & $\mathrm{X}$ \\
\hline VOCHYSIACEAE & Qualea gestasiana A.St.-Hil. */*** & $\begin{array}{l}7590 \\
7591 \\
7596 \\
7601 \\
7610\end{array}$ & $\mathrm{x}$ & $\mathrm{X}$ \\
\hline WINTERACEAE & Drimys brasiliensis Miers * & $\begin{array}{l}8775 \\
8947\end{array}$ & & $\mathrm{X}$ \\
\hline
\end{tabular}

Foram confeccionados corpos de provas orientados em plano transversal, os quais foram polidos com lixas d'água com granulometria decrescente (80 a 1.200). A análise de características anatômicas macroscópicas foi realizada com olhos desarmados e com lupa conta-fios com aumento de 10 vezes. As imagens foram capturadas utilizando microscópio estereoscópico Leica, modelo MZ16, acoplado a uma câmera Leica ligada a um computador com o software Image Manager (IM50), ou microscópio estereoscópico Olympus SZX12, acoplado à câmera Sony Cyber Shot com 8.1 Megapixels de resolução. As descrições macroscópicas da madeira seguiram as características propostas por Ruffinatto et al. (2015).
Para a análise de características microscópicas, foram utilizadas lâminas permanentes do acervo da RBw, confeccionadas pelo pesquisador Cunha Mello na década de 1950, a partir de amostras de madeira provenientes do PARNA Itatiaia. A metodologia de confecção das lâminas permanentes foi descrita por Barros et al. (2008a), utilizando de 1 a 5 amostras para cada uma das 13 espécies que foram analisadas e descritas (Tabela 1). A captura de imagens foi realizada com a câmera digital Olympus DP73, acoplada a um microscópio Olympus BX50 e analisada no software Image Pro-Plus (4.0, versão para Windows). As descrições microscópicas 
e mensurações dos elementos celulares obedeceram às normas propostas em IAWA Committee (1989).

Duas chaves interativas de identificação foram desenvolvidas na plataforma Xper3, de domínio livre e disponível em <http:/www. xper3.com/>. O software Xper3 foi desenvolvido pelo Laboratório de Informática e Sistemática da Universidade Pierre et Marie Curie (Paris), membro da UMR 7.205 (CNRS, MNHN, UPMC), em 1991. Seu uso é gratuito, e o usuário deve ter a licença permitida pela Creative Commons Attribution-Share Alike 3.0 França. Esse software possui aplicação executada por navegador de internet, sendo possível o gerenciamento de dados colaborativos, armazenando e editando dados descritivos. Seu uso permite a criação de um ou mais bancos de dados que possibilita o desenvolvimento de chaves de identificação, matrizes de comparação de todas as espécies e caracteres, além da seleção de análises entre espécies e/ou grupos.

Para o desenvolvimento das chaves de identificação, foram cadastradas as características anatômicas e os táxons, e foi realizada a associação entre as características e os táxons. Também foram inseridas imagens anatômicas associadas aos táxons.

A chave de identificação baseada em caracteres macroscópicos, intitulada "Chave de identificação macroscópica de madeiras do PARNA do Itatiaia, RJ", contou com a inserção de 41 espécies. As descrições que embasaram o desenvolvimento da chave foram provenientes deste estudo (13 espécies) e do banco de dados do Laboratório de Botânica Estrutural do Instituto Jardim Botânico do Rio de Janeiro (28 espécies) (Tabela 1).

A chave de identificação baseada em caracteres microscópicos, intitulada "Chave de identificação microscópica de madeiras do PARNA do Itatiaia, RJ", contou com a inserção de 61 espécies. As descrições que embasaram o desenvolvimento da chave foram provenientes do presente trabalho (13 espécies), do estudo de Barros et al. (2008b) (29 espécies) e do banco de dados do Laboratório de Botânica Estrutural do Instituto Jardim Botânico do Rio de Janeiro (19 espécies) (Tabela 1).
Informações sobre o status de conservação foram obtidas no site do Herbário Virtual Reflora, que conta com os registros, imagens e outras informações relacionadas às coleções do Herbário Jardim Botânico e de outros acervos parceiros, de instituições nacionais $e$ internacionais; $e$ os nomes populares, nas Fichas de Características das Madeiras Brasileiras (IPT 1989).

\section{Resultados}

A seguir, as descrições macro e microscópicas das espécies analisadas, relacionadas em ordem alfabética.

\section{ANNONACEAE}

\section{Guatteria candolleana Schltdl.}

Números dos caracteres macroscópicos, segundo Ruffinatto et al. (2015): 3p, 12p, 16p, 20p, 26p, 40p, 42p (scalariform), 43p (rays visible), $44 \mathrm{p}$ (rays visible), 45p (larger rays as wide or wider than wider pores), $47 p$ (not storied), 48p (highest rays less $5 \mathrm{~mm}$ high), 49p ( $\leq 4 / \mathrm{mm}$ ), 60p, 68p, 94p. (Figura 1: F).

Números dos caracteres microscópicos do IAWA (1989): 1p, 5p, 12p, 13p, 22p, 26p, 30p, 42p, 47p, 53p, 61p, 66p, 68p, 72p, 76p, 79p, 88p, 92p, 98p, 102p, 103p, 107p, 114p, 183p, 186p, 187p, 189p, 197p. (Figura 2: A-C).

\section{BIGNONIACEAE}

\section{Jacaranda caroba (Vell.) A. DC.}

Números dos caracteres macroscópicos, segundo Ruffinatto et al. (2015): $1 p, 2 p(6)$, 3p, 12p, 17p, 19p, 31p, 42p (vasicentric), 43p (ray visible), 44p (rays visible), 45p (larger rays narrower than wider pores), 47p (not storied), 48p (highest rays less than $5 \mathrm{~mm}$ high), 49p (>12/mm), 60p, 68p, 94p. (Figura 1: G).

\section{Números dos caracteres microscópicos} do IAWA (1989): 1p, 5p, 13p, 22p, 23p, 25p, 31p, 41p, 48p, 53p, 61p, 66p, 68p, 71p, 76p, 79p, 80p, 81p, 83p, 91p, 92p, 97p, 107p, 116p, 183p, 186p, 187p, 189p, 197p. (Figura 2: D-F). 


\section{CLETHRACEAE}

\section{Clethra scabra var. reticulata (Meisn.) Sleumer}

Números dos caracteres macroscópicos, segundo Ruffinatto et al. (2015): $1 p, 2 p$ (10), 3p, 13p, 18p, 19p, 43p (rays not visible), $44 p$ (rays not visible), 45 (larger rays narrower than wider pores), 47p (not storied), 48p (highest rays less $5 \mathrm{~mm}$ high), 49p (5-12/mm), 60p, 68p, 94p. (Figura 1: B).

Números dos caracteres microscópicos do IAWA (1989): 1p, 5p, 9p, 12p, 14p, 17p, 22p, 23p, 25p, 30p, 41p, 49p, 53p, 61p, 66p, 69p, 71p, 76p, 78p, 91p, 92p, 93p, 98p, 107p, 115p, 183p, 186p, 187p, 189p, 197p.

(Figura 2: G-I).

\section{EUPHORBIACEAE}

\section{Croton floribundus Spreng.}

Números dos caracteres macroscópicos, segundo Ruffinatto et al. (2015): $1 p, 2 p$ (4), 3p, 12p, 17p, 19p, 26p, 43p (rays visible), $44 p$, (rays visible), 45p (larger rays narrower than wider pores), 47p (not storied), 48p (highest rays less $5 \mathrm{~mm}$ high), 49p (5-12/mm) 60p, 68p, 94p. (Figura 1: C).

Números dos caracteres microscópicos do IAWA (1989): 2p, 5p, 13p, 22p, 25p, 31p, 41p, 47p, 53p, 61p, 65p, 66p, 68p, 71p, 76p, 92p, 97p, 98p, 108p, 115p, 183p, 186p, 187p, 189p, 197p. (Figura 2: A-C).

\section{Croton vulnerarius Baill.}

Números dos caracteres macroscópicos, segundo Ruffinatto et al. (2015): $1 p, 2 p$ (4), 3p, 12p, 17p, 20p, 42p (absent), 43p (rays visible), 44p (rays visible) 45p (larger rays narrower than wider pores), 47p (not storied), $48 \mathrm{p}$ (highest rays less than $5 \mathrm{~mm}$ high), 49p (>12/mm) 60p, 68p, 94p. (Figura 1: D).

Números dos caracteres microscópicos do IAWA (1989): 1p, 5p, 13p, 22p, 25p, 30p, 41p, 47p, 53p, 61p, 66p, 69p, 71p, 76p, 79p, 91p, 92p, 93p, 96p, 106p, 107p, 116p, 183p, 186p, 187p, 189p, 197p. (Figura 2: M-O).

\section{FABACEAE}

\section{Dalbergia variabilis Vogel}

Números dos caracteres macroscópicos, segundo Ruffinatto et al. (2015): $1 p, 2 p$ (4), 4p, 12p, 17p, 20p, 26p, 30p, 31p, 32p, $34 \mathrm{p}, 38 \mathrm{p}, 42 \mathrm{p}$ (confluent), 43p (rays not visible), $44 p$ (rays not visible), 45p (larger rays narrower than wider pores), 47p (regular fine storying), $48 p$ (highest rays less than $5 \mathrm{~mm}$ high), 49p (>12/mm), 60p, 68p, 94p. (Figura 1: E).

Números dos caracteres microscópicos do IAWA (1989): 1p, 4p, 13p, 22p, 25p, 30p, 42p, 47p, 53p, 61p, 66p, 68p, 72p 76p, 77p, 79p, 80p, 81p, 83p, 89p, 91p, 92p, 93p, 97p, 104p, 116p, 118p, 120p, 136p, 142p, 183p, 186p, 187p, 189p, 197p. (Figura 3: A-C).

\section{Leucochloron incuriale (Vell.) Barneby \& J.W. Grimes}

Números dos caracteres macroscópicos, segundo Ruffinatto et al. (2015): $1 p, 2 p$ (6), 3p, 12p, 17p, 20p, 26p, 32p, 34p, 38p, $42 \mathrm{p}$ (confluent), 43p (rays not visible), 44p (rays not visible), 45p (larger rays narrower than wider pores), 47p (not storied), 48p (highest rays less $5 \mathrm{~mm}$ high), 49p (>12/mm) 60p, 68p, 94p. (Figura 1: H).

Números dos caracteres microscópicos do IAWA (1989): 1p, 5p, 13p, 22p, 25p, 30p, 42p, 47p, 53p, 61p, 66p, 68p, 71p, 80p, 81p, 83p, 89p, 91p, 92p, 97p, 104p, 116p, 186p, 187p, 189p, 197p. (Figura 2: D-F).

\section{Lonchocarpus sericeus (Poir.) Kunth ex DC.}

Números dos caracteres macroscópicos, segundo Ruffinatto et al. (2015): $1 p, 2 p$ (4), 3p, 12p, 17p, 20p, 26p, 31p, 32p, 34p, $38 p, 42 p$ (confluent), 43p (rays not visible), 44p (rays not visible), 45p (larger rays narrower than wider pores), 48p (highest rays less $5 \mathrm{~mm}$ high), 49p (>12/mm), 60p, 68p, 94p. (Figura 1: I).

Números dos caracteres microscópicos do IAWA (1989): 1p, 5p, 13p, 22p, 25p, 30p, 42p, 46p, 52p, 61p, 66p, 69p, 72p, 79p, 80p, 81p, 83p, 85p, 89p, 91p, 97p, 104p, 116p, 118p, 120p, 136p, 142p, 183p, 186p, 187p, 189p, 197p. (Figura 3: G-I). 


\section{Machaerium nyctitans (Vell.) Benth.}

Números dos caracteres macroscópicos, segundo Ruffinatto et al. (2015): $1 p, 2 p$ (11), 3p, 12p, 17p, 19p, 26p, 30p, 32p, 34p, 35p (majory narrow), 38p, 42p (lozenge-aliform), 43p (rays not visible), 44p (rays not visible), 45p (larger rays narrower than wider pores), 47p (storied), 48p (highest rays less than $5 \mathrm{~mm}$ high), 49p (>12/mm), 60p, 68p, 94p. (Figura 1: J).

Números dos caracteres microscópicos do IAWA (1989): 1p, 5p, 7p, 13p, 22p, 27p, 30p, 42p, 47p, 53p, 61p, 66p, 69p, 71p, 77p, 79p, 80p, 83p, 86p, 89p, 92p, 97p, 104p, 116p, 118p, 120p, 136p 142p, 183p, 186p, 187p, 189p, 197p. (Figura 3: J-L).

\section{Peltophorum vogelianum Benth.}

Números dos caracteres macroscópicos, segundo Ruffinatto et al. (2015): $1 p, 2 p$ (3), 3p, 12p, 17p, 20p, 31p, 32p, 34p, 38p, $42 p$ (vasicentric), 43p (all rays visible), $44 p$ (rays visible), 45p (larger rays narrower than pores), $47 p$ (not storied), 48p (highest rays less than 5 millimetres hight), 49p (>12/mm), 60p, 68p, 94p. (Figura 1: K).

Números dos caracteres microscópicos do IAWA (1989): 1p, 5p, 13p, 22p, 25p, 30p, 42p, 46p, 53p, 61p, 68p, 71p, 79p, 80p, 81p, 83p, 89p, 91p, 96p, 104p, 116p, 186p, 187p, 189p, 197p. (Figura 3: M-O).

\section{Senna multijuga (Rich.) H.S.Irwin \& Barneby}

Números dos caracteres macroscópicos, segundo Ruffinatto et al. (2015): $1 p, 2 p$ (4), 3p, 12p, 16p, 20p, 26p, 32p, 34p, 38p, $42 \mathrm{p}$ (lozange-aliform), 43p (rays not visible), $44 p$ (rays not visible), 45p (Larger rays narrower than wider pores) $47 \mathrm{p}$ (not storied), 48p (highest rays less than 5 millimetres hight), 49p (5-12/mm), 60p, 68p, 94p. (Figura 1: L).

Números dos caracteres microscópicos do IAWA (1989): $1 p, 5 p, 13 p, 22 p, 25 p$, 30p, 42p, 46p, 53p, 61p, 66p, 69p, 72p, 80p, 81p, 83p, 84p, 89p, 91p, 97p, 104p, 115p, 136p, 142p, 183p, 186p, 187p, 189p, 197p. (Figura 4: A-C).

\section{LAMIACEAE}

\section{Vitex taruma Mart.}

Números dos caracteres macroscópicos, segundo Ruffinatto et al. (2015): $1 p, 2 p(2)$, 3p, 12p, 17p, 19p, 31p, 42p(vasicentric), 43(rays not visible), 44p (rays not visible), 45p (larger rays wide or wider than wider pores), $47 \mathrm{p}$ (not storied), 48p (highest rays less than $5 \mathrm{~mm}$ high), 49p (5-12/mm), 60p, 68p, 94p. (Figura 1M).

Números dos Caracteres Microscópicos do IAWA (1989): 1p, 5p, 13p, 21p, 25p, 31p, 41p, 47p, 53p, 61p, 66p, 69p, 72p, 78p, 79p, 92p, 97p, 98p, 106p, 107p, 115p, 183p, 186p, 187p, 189p, 197p. (Figura 4: D-F).

\section{MELIACEAE}

\section{Cabralea canjerana (Vell.) Mart.}

Números dos caracteres macroscópicos segundo Ruffinatto et al . (2015): $1 p, 2 p(7)$, 3p, 12p, 17p, 19p, 26p, 35p (majority wide), $42 p$ (banded), 43p (rays not visible), 44p (rays not visible), 45p (larger rays narrower than wider pores), 47p (not storied), 48p (highest rays less than $5 \mathrm{~mm}$ high), 49p (>12/mm), 60p, 68p, 94p. (Figura 1: A).

Números dos caracteres microscópicos do IAWA (1989): $1 p, 5 p, 13 p, 22 p, 24 p$, 30p, 42p, 47p, 53p, 61p 66p, 69p, 72p, 85p, 91p, 92p, 97p, 107p, 116p, 183p, 186p, 187p, 189p, 197p. (Figura 4: G-I).

As chaves interativas intituladas "Chave de identificação macroscópica de madeiras do PARNA do Itatiaia, RJ" e "Chave de identificação microscópica de madeiras do PARNA do Itatiaia, RJ" estão disponíveis no site do Laboratório de Anatomia da Madeira e Dendrocronologia (LAMAD), hospedado no site da Universidade Federal Fluminense (UFF), em: <http://gbg.sites. uff.br/lamad/> (Figura 5). 

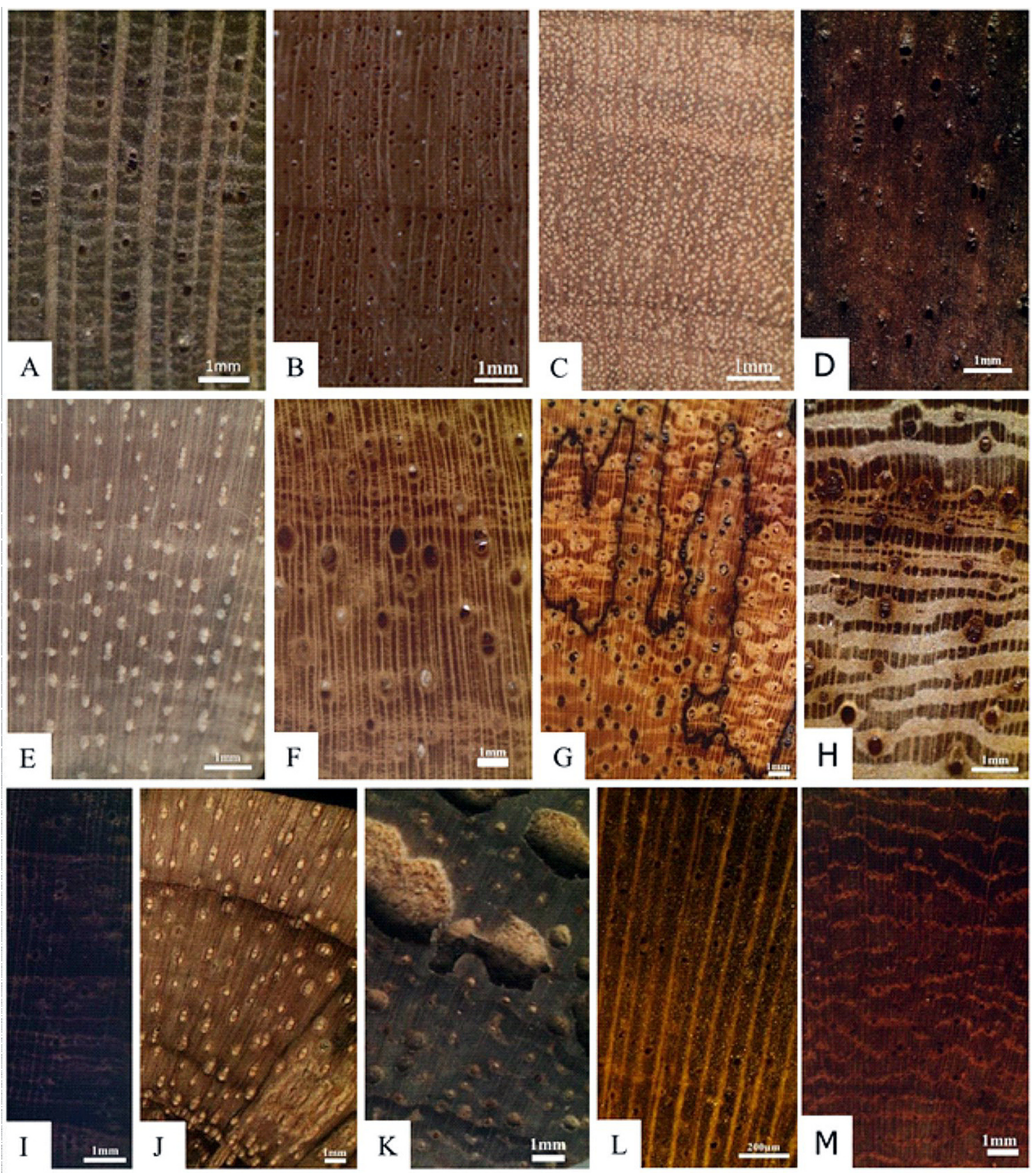

Figura 1 - Fotografias macroscópicas, secção transversal do caule. A: Guatteria candolleana; B: Jacaranda caroba; C: Clethra scabra var. reticulata; D: Croton floribundus; E: Croton vulnerarius; F: Dalbergia variabilis; G: Leucochloron incuriale; H: Lonchocarpus sericeus; I: Machaerium nyctitans; J: Peltophorum vogelianum; K: Senna multijuga; L: Vitex taruma; M: Cabralea canjerana. 


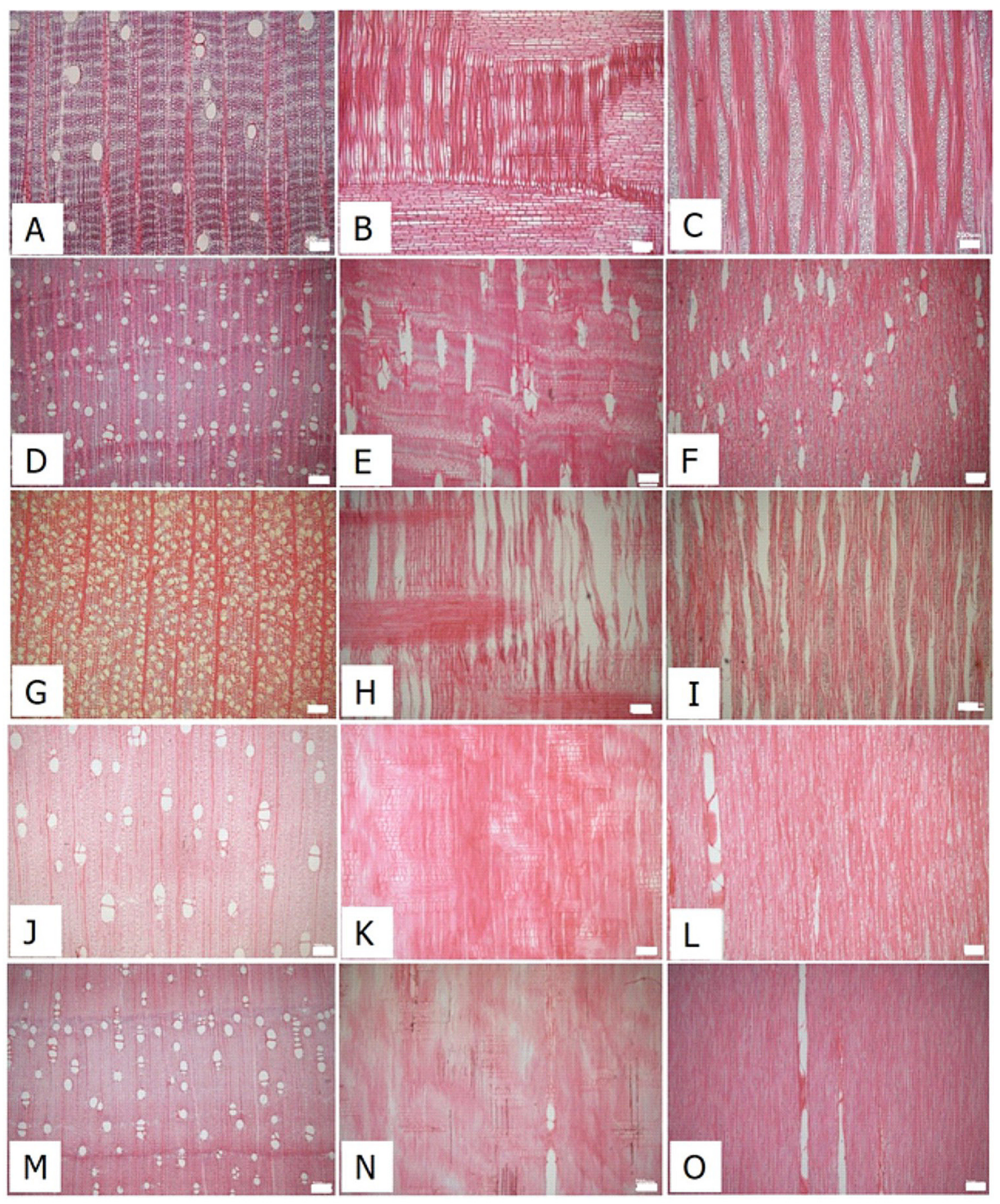

Figura 2 - Fotografias microscópicas, secções transversal, longitudinal radial e longitudinal tangencial de caules. A-C: Guatteria candolleana; D-F: Jacaranda caroba; G-I: Clethra scabra var. reticulate; J-L: Croton

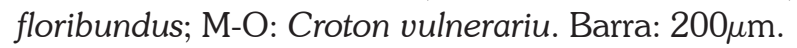




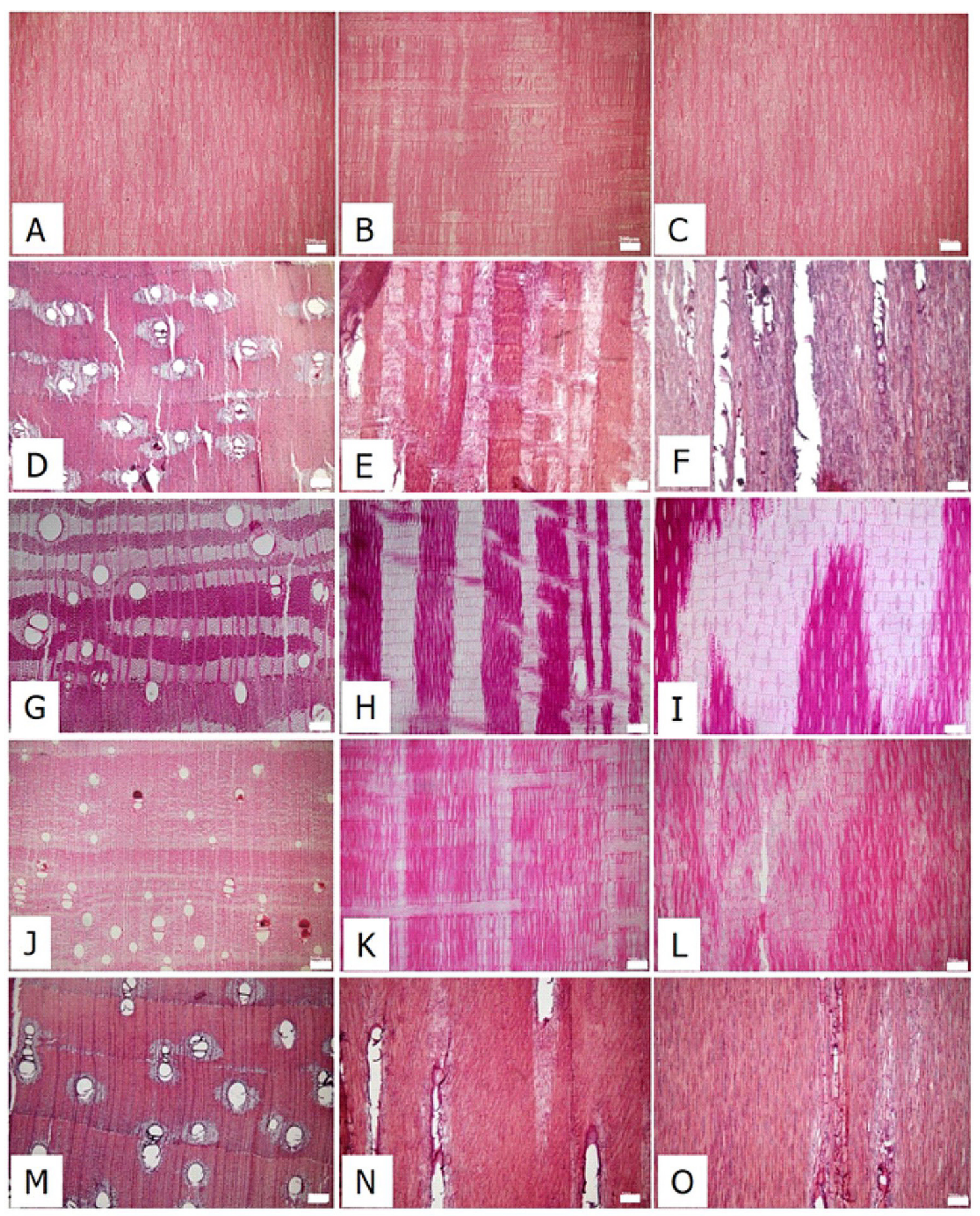

Figura 3 - Fotografias microscópicas, secções transversal, longitudinal radial e longitudinal tangencial de caules. A-C: Dalbergia variabilis; D-F: Leucochloron incuriale; G-I: Lonchocarpus sericeus; J-L: Machaerium nyctitans; M-O: Peltophorum vogelianum. Barra: $200 \mu \mathrm{m}$. 


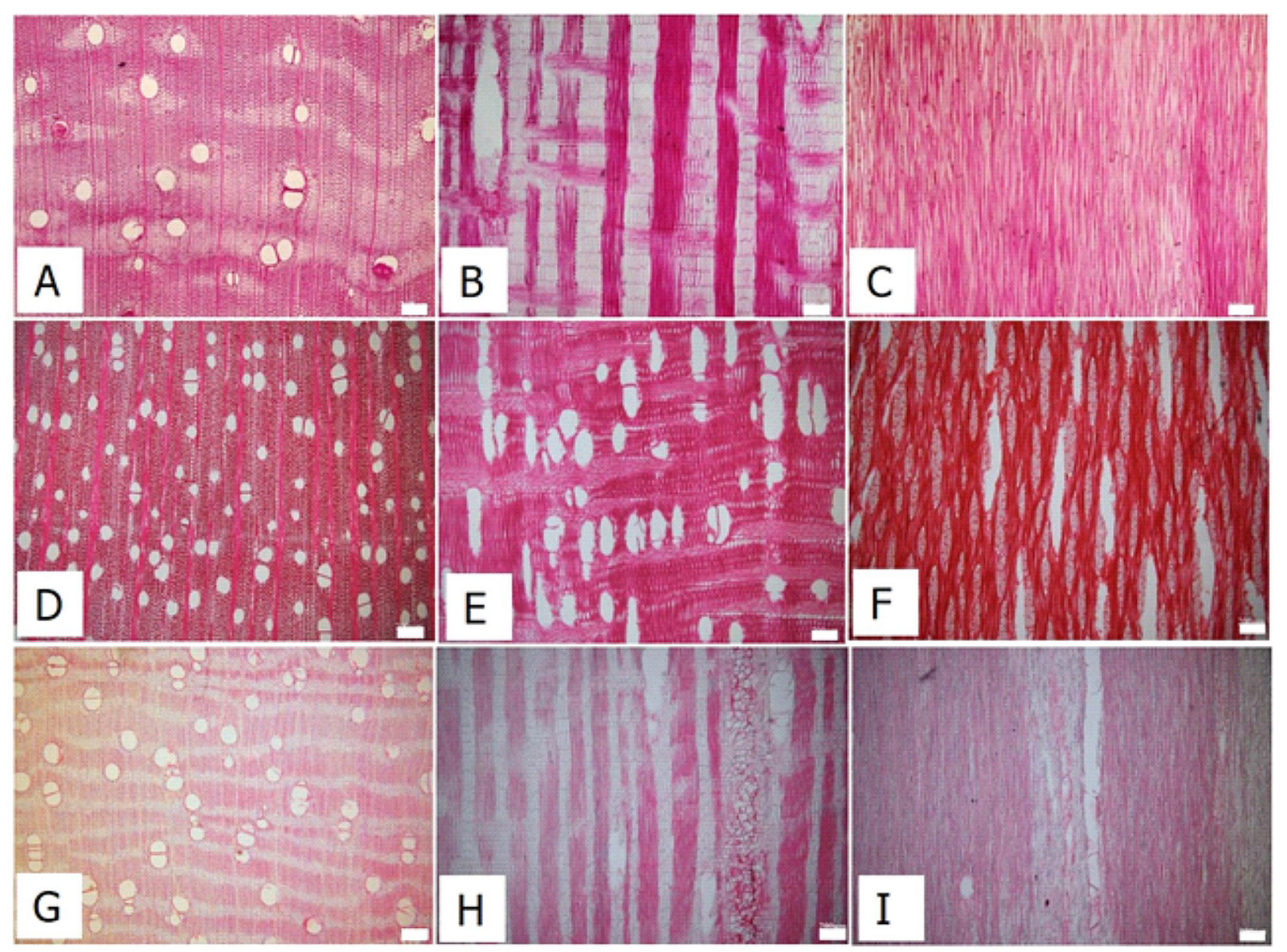

Figura 4 - Fotografias microscópicas, secções transversal, longitudinal radial e longitudinal tangencial. A-C: Senna multijuga; D-F: Vitex trauma; G-I: Cabralea canjerana. Barra: $200 \mu \mathrm{m}$. 


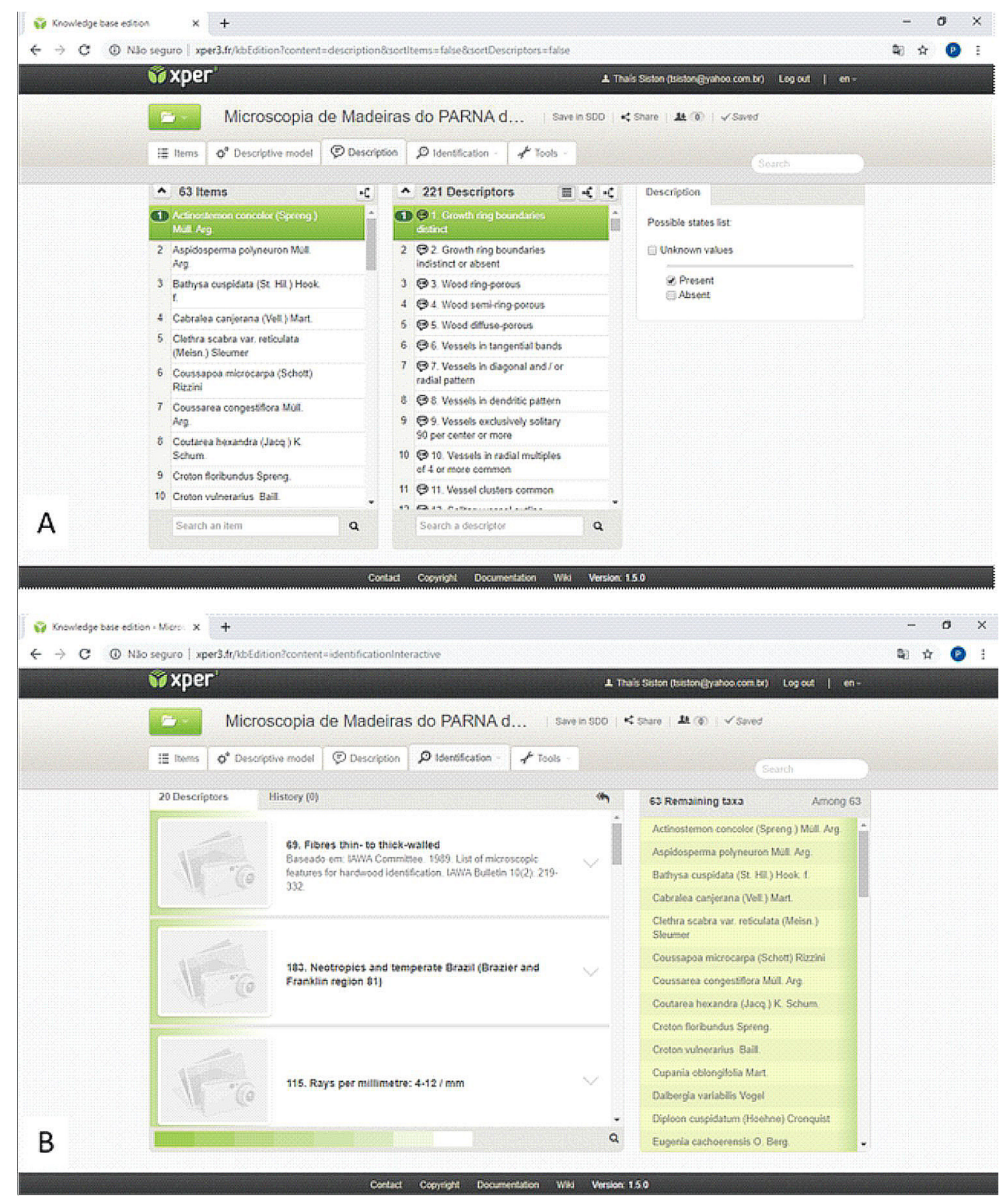

Figura 5 - Software Xper3. A: Correlação caracteres x espécie; B: Chave.

\section{Discussão}

As chaves interativas são muito versáteis quando comparadas às chaves tradicionais de identificação, e apresentam inúmeras vantagens na confecção e no uso, a saber: disponibilização $e$ atualização da chave; inserção e modificação dos caracteres inseridos no software, inclusão de informações e observações adicionais; agilidade no manuseio; possibilidade de entrada de múltiplos caracteres e sem necessidade de ordem pré-definida; e possibilidade de comparação entre táxons (Dallwitz et al. 2000, Brach \& Song 2005, Bittrich et al. 2012, Espírito Santo et al. 2013,
Vergne 2014). Alguns aspectos negativos para o uso dessas chaves interativas estão relacionados com a obrigatoriedade do uso de computadores e de internet, o que pode dificultar a utilização em locais mais isolados.

O software Xper3 foi escolhido após o teste de outros softwares, como o Delta, igualmente gratuito e de acesso livre. A decisão por esse software ocorreu devido a alguns pontos relevantes, como a segurança na compatibilidade com os tipos de navegadores de internet mais utilizados e a não necessidade de baixar o software para o computador. Outros pontos importantes foram a 
fácil utilização, com gráficos bastante amigáveis e intuitivos, e a hospedagem de todas as informações em um mesmo login. Por fim, a atualização do software, a disponibilização de manuais de uso $e$ o suporte ao usuário são extremamente eficazes.

As chaves desenvolvidas neste trabalho, em plataforma Xper3, são as primeiras chaves interativas com caracteres macroscópicos $e$ microscópicos da madeira para o bioma Mata Atlântica, além de seguirem a padronização dos caracteres tanto para macroscopia quanto para microscopia, Rufinatto et al. (2015) e IAWA (1989), respectivamente. Coradin et al. (2010) desenvolveram uma chave interativa para identificação macroscópica de madeiras comerciais brasileiras em plataforma Delta, e a escolha dos caracteres foi feita com base nas recomendações de Coradin \& Muniz (1992) e em outras descrições macroscópicas publicadas até o momento. O uso de padrões para descrição anatômica auxilia no processo de identificação de madeiras, permitindo melhor troca de informações e traduções, simplificando e padronizando a elaboração de ferramentas de identificação.

Além da chave macroscópica de Coradin et al. (2010), Richter et al. (2002) publicaram uma chave em Delta para identificação microscópica das madeiras comerciais utilizadas no comércio internacional. Até o momento, essas eram as únicas chaves interativas disponíveis para os especialistas em anatomia da madeira e para os técnicos que realizam identificação forense (Dormont et al. 2015). Como mencionado, as duas publicações tratam de madeiras comerciais, enquanto as chaves ora apresentadas relacionam espécies arbóreas de uma importante unidade de conservação brasileira. Essa peculiaridade permite a identificação das madeiras do PARNA do Itatiaia, a divulgação das árvores da unidade de conservação - sob a perspectiva de suas madeiras -, e sua utilização como material didático e de divulgação, auxiliando na formação de recursos humanos na área da biodiversidade.

\section{Conclusão}

As madeiras coletadas no PARNA Itatiaia foram descritas macroscópicas e microscopicamente, permitindo aumentar o banco de dados de informações de espécies do Parque e da Mata Atlântica. Em conjunto com a compilação de dados de publicações e da base de dados do
LBE, foi possível construir uma grande matriz e desenvolver as chaves interativas Macroscopia e Microscopia do PARNA Itatiaia. É importante ressaltar que essas são as primeiras chaves macroscópica e microscópica para espécies do bioma Mata Atlântica e para uma unidade de conservação brasileira.

Portanto, o estudo contribui com as metas propostas na Convenção sobre Diversidade Biológica, que englobam o conhecimento, descrição, publicação e divulgação dos dados relacionados às unidades de conservação brasileiras e aos seres vivos, em especial as espécies arbóreas, que integram esse ecossistema.

\section{Agradecimentos}

Os autores agradecem ao Instituto de Pesquisas Jardim Botânico do Rio de Janeiro e à Universidade Federal Fluminense, pelo suporte de laboratórios; à Escola de Botânica Tropical, Programa de Pós-Graduação Stricto Sensu em Botânica Diversidade Vegetal: Conhecer e Conservar, por meio do Mestrado Profissional. Aos órgãos FAPERJ, CAPES e CNPq, pelas bolsas e projetos financiado e apoiado.

\section{Referências Bibliográficas}

Barros CF, Coradin VTR. 2015. Redes de Herbários e Herbários Virtuais do Brasil. In: $66^{\circ}$ Congresso Nacional de Botânica. UNISANTA Bioscience - Edição Especial, 4(7), 2015.

Barros CF, Callado CH, Cunha M, Ferreira MLM, Tamaio NM, Costa OCG. 2008. Madeiras da Mata Atlântica: Anatomia do lenho de espécies ocorrentes nos remanescentes florestais do Estado do Rio de Janeiro. 1 ed. Rio de Janeiro: Instituto de Pesquisas Jardim Botânico do Rio de Janeiro. vol. III. 103p.

Bittrich V, Souza CSD, Coelho RLG, Martins MV, Hopkins MJG, Amaral MCE. An interactive key (Lucid) for the identifying of the genera of seed plants from the Ducke Reserve, Manaus, AM, Brasil. Rodriguésia, 63:55-64, 2012.

Brach AR, Song H. ActKey: a web-based interactive identification key program. Taxon, 54: 1041-1046, 2005.

Brandes AFN, Barros CF. Anatomia do lenho de oito espécies de lianas da família Leguminosae ocorrentes na Floresta Atlântica. Acta Botânica Brasílica (Impresso), 22: 465-480, 2008. 
Brandes AFN, Lisi CS, Barros CF. Dendrochronology of lianas of the Leguminosae family from the Atlantic Forest, Brazil. Trees, 25: 133-144, 2011.

Brasil 2000. Lei $n^{\circ}$ 9.985, de 18 de julho de 2000. Diário Oficial da União. <http://www.planalto.gov.br/ ccivil_03/LEIS/L9985.htm > . Acesso em: 15/03/2016.

Brasil 1990. Decerto $n^{\circ} 99.547$, de 25 de setembro de 1990. Diário Oficial da União. <https://www2. camara.leg.br/legin/fed/decret/1990/decreto-9954725-setembro-1990-339012-publicacaooriginal-1-pe. html > Acesso em: 21/04/2014.

Brasil 2006. Lei $\mathrm{n}^{\circ} 11.428$, de 22 de dezembro de 2006. Diário Oficial da União. <http://www.planalto. gov.br/ccivil_03/_Ato2004-2006/2006/Lei/L11428. $\mathrm{htm}>$. Acesso em: 15/03/2016.

Camargo JAA, Coradin VTR, Czarneski CM, Oliveira $\mathrm{D}$ de, Meguerditehian I. 2001. Catálogo de árvores do Brasil. Instituto Brasileiro do meio Ambiente e dos Recursos Naturais Renováveis, Laboratório de Produtos Florestais. Ed. IBAMA. 896p.

Campanili M, Schaffer WB (orgs.). 2010. Mata Atlântica: patrimônio nacional dos brasileiros/ Ministério do Meio Ambiente. Secretaria de Biodiversidade e Florestas. Núcleo Mata Atlântica e Pampa. MMA. 408p.

CNUC/MMA. Consulta por UC: Parque Nacional do Itatiaia. <http:/www.mma.gov.br/areas-protegidas/ cadastro-nacional-de-ucs/consulta-por-uc $>$. Acesso em: 21/04/2014.

Coradin VTR, Muñiz GIB. 1992. Normas de procedimentos em estudos de anatomia da madeira: I-Angiospermae, II-Gimnospermae. Laboratório de Produtos Florestais, Série Técnica 15.

Coradin VTR, Camargos JAA, Pastore TCM, Christo AG. 2010. Madeiras comerciais do Brasil: chave interativa de identificação baseada em caracteres gerais $e$ macroscópicos $=$ Brazilian commercial timbers: interactive identification key based on general and macroscopic features. Serviço Florestal Brasileiro, Laboratório de Produtos Florestais. CD-ROM.

Cunha ME. 1950. Estudo dendrológico de essências florestais do Parque Nacional de Itatiaia e os caracteres anatômicos de seus lenhos. Boletim do Parque Nacional do Itatiaia, Ministério da Agricultura Serviço Florestal, n. 2. 172p.

Dallwitz M, Pankhurst R. 1988. DELTA Newsletter 1. Originally published in hard copy. This reformatted electronic version is available at http://delta-intkey. com.

Dallwitz MJ, Paine TA, Zurcher EJ. 2000. Principles of interactive keys. $<$ http://delta-intkey.com $>$. Acesso em: 17/12/2016.
Dormont EE et al. Forensic timber identification: It's time to integrate disciplines to combat ilegal logging. Biological Conservation, 191:790-798, 2015.

Espírito Santo FS, Siqueira AA, Rapini A. Chave interativa para a identificação das espécies da Aliança Tabebuia (Bignoniaceae) no estado da Bahia, Brasil. Biota Neotrop., 13(3): 345-349, 2013.

Espírito Santo FS, Silva-Castro MM, Rapini A. 2013. Flora da Bahia: Bignoniaceae 2 - Aliança Tabebuia. Sitientibus Série Ciências Biológicas, 13. <http:// pkp.uefs.br/ojs/index.php/sitientibusBiologia/article/ view/211/282 > . Acesso em: 26/07/2016.

Flora do Brasil 2020, em construção. Jardim Botânico do Rio de Janeiro. < http://floradobrasil.jbrj.gov.br/ > . Acesso em: 16/10/2019.

Flora do Brasil 2020, em construção. Reflora. Jardim Botânico do Rio de Janeiro. <http://floradobrasil.jbrj. gov.br/> . Acesso em: 16/10/2019.

Forzza RC, Baumgratz JFA, Bicudo CEM, Canhos DAL, Carvalho Júnior AA, Nadruz Coelho MA, Costa AF, Costa DP, Hopkins MG, Leitman PM, Lohmann LG, Lughadha EM, Maia LC, Martinelli G, Menezes M, Morim MP, Peixoto AL, Pirani JR, Prado J, Queiroz LP, Souza S, Souza VC, Stehmann JR, Sylvestre LS, Walter BMT \& Zappi DC. New Brazilian Floristic List Highlights Conservation Challenges. BioScience, 62(1): 135-159, 2012.

Fundação SOS Mata Atlântica, Instituto Nacional de Pesquisas Espaciais, 2018. Atlas dos Remanescentes Florestais da Mata Atlântica - Período 2016-2017. Relatório Técnico. <http://mapas.sosma.org.br/site media/download/Atlas_Mata_Atlantica_20162017_ relatorio_tecnico_2018_final.pdf $>$. Acesso em: 17/10/2019.

IAWA Committee. IAWA List of Microscopic Features for Hardwood Identification. IAWA, 10(2): 219-332, 1989 .

ICMBio. PARNA do Itatiaia. 2014b. <http://www. icmbio.gov.br/portal/biodiversidade/unidades-deconservacao/biomas-brasileiros/mata-atlantica/ unidades-de-conservacao-mata-atlantica/2181-parnado-itatiaia.html > . Acesso em: 21/04/2014.

ICMBio. Unidades de Conservação: Mata Atlântica. 2014a. http://www.icmbio.gov.br/portal/biodiversidade/ unidades-de-conservacao/biomas-brasileiros/mataatlantica/unidades-de-conservacao-mata-atlantica. Acesso em: 19/04/2014.

Instituto de Pesquisas Tecnológicas do Estado de São Paulo - IPT. 1989. Fichas de Características das Madeiras Brasileiras. 2 ed. IPT. 418p. 
JBRJ - Instituto de Pesquisas Jardim Botânico do Rio de Janeiro. Jabot - Banco de Dados da Flora Brasileira. 2016. <http://www.jbrj.gov.br/jabot>. Acesso em: $12 / 10 / 2016$.

Koch G, Richter HG, Schmitt U. Design and application of citeswoodID computer-aided identification and description of cites-protected timbres. IAWA Journal, 32(2): 213-220, 2011.

Mainieri C. 1958. Identificação das principais madeiras de comércio no Brasil. Boletim Instituto de Pesquisas Tecnológicas, 686: 39.

Ministério do Meio Ambiente. 2008. Instrução Normativa $n^{\circ} 6$, de 23 de setembro de 2008. Diário Oficial da União. <www.ibama.gov.br/phocadownload/ category/47-_?download $=999 \% 3$ A_06-2008.p $>$. Acesso em: 11/06/2016.

Mittermeier RA, Gil RP, Hoffman M, Pilgrim Brooks JT, Mittermeier CG, Lamoreux J, Fonseca GAB. 2005. Hotspots revisited: Earth's biologically richest and most endangered terrestrial ecoregions. University of Chicago Press. 392p.

Moreira FGL. 2017. Chave de identificação interativa para as espécies da ordem Gentianales em um fragmento de Mata Atlântica no campus central-UFRN. Trabalho de Conclusão de Curso. Universidade Federal do Rio Grande do Norte. 46p.

Myers N, Mittermeier RA, Mittermeier CG, Da Fonseca GAB, Kent J. Biodiversity hotspots for conservation priorities. Nature, 403: 853-858, 2000.

Record SJ. Keys to American woods (continued). Trop. Woods, 77: 18-38, 1994.

Ribeiro MC, Martensen AC, Metzger JP, Tabarelli M, Scarabo F, Fortin MJ. 2011. The Brazilian Atlantic Forest: a shrinking biodiversity hotspot, p. 405-434.
In: Zachos FE, Habel JC (eds.). Biodiversity Hotspots: distribution and protection of conservation priority areas. Heidelbelberg, Springer.

Richter HG, Oelker M, Krämer G. 2002. MacroHOLZdata - Computer-gestützte makroskopische Holzartenbestimmung sowie Informationen $\mathrm{zu}$ Eigenschaften und Verwendung von Nutzhölzern. CDROM, Holzfachschule Bad Wildungen, Eigenverlag.

Rodrigues CGO, Tomzhinski GW, Irving MA, Correa FV. 2013. Parque Nacional do Itatiaia: o primeiro parque nacional do Brasil, p. 79-108. In: Marta de Azevedo Irving; Frances Vivian Corrêa ACZ (org.). Parques Nacionais do Rio de Janeiro: desafios para uma gestão social da biodiversidade. 1 ed. Letra e Imagem.

Ruffinatto F, Crivellaro A, Wiedenhoeft AC. Macroscopic wood features list. IAWA Journal. 36(2): 208-241, 2015.

Scarano FR, Ceotto P. Brazilian Atlantic forest: impact, vulnerability, and adaptation to climate change. Biodivers Conserv. 24: 2319-2331, 2015.

Silva Neto SJ, Avila Jr RS. Uma nova espécie de Randia (Rubiaceae, Gardenieae) para o estado do Rio de Janeiro, Brasil. Rodriguesia, 58(4): 739-742, 2007.

SOS Mata Atlântica Online. 2019. Mata Atlântica. <https://www.sosma.org.br/conheca/mata-atlantica/>. Acesso em: 16/10/2019.

Vergne MC. 2014. Chave de identificação interativa de múltiplas entradas para espécies da família Lauraceae da Reserva Natural Vale, Linhares-ES/Matheus Carvalho Vergne. 77p.

Wolowski M, Freitas L. 2015. Biologia reprodutiva e interações planta-polinizador no Parque Nacional do Itatiaia. Boletim do Parque Nacional do Itatiaia: 20.

Biodiversidade Brasileira - BioBrasil.

Edição temática: Diálogos entre a Academia e a Gestão de Áreas Protegidas:

Programa de Pós-Graduação Profissional - Biodiversidade em Unidades de Conservação

n. 2, 2020

http://www.icmbio.gov.br/revistaeletronica/index.php/BioBR

Biodiversidade Brasileira é uma publicação eletrônica científica do Instituto Chico Mendes de

Conservação da Biodiversidade (ICMBio) que tem como objetivo fomentar a discussão e a disseminação de experiências em conservação e manejo, com foco em unidades de conservação e espécies ameaçadas.

ISSN: 2236-2886 